\title{
Prevalencia y características de la enfermedad pulmonar obstructiva crónica en la República de Panamá
}

\author{
Prevalence and characteristics of chronic obstructive \\ pulmonary disease in Panama Republic
}

\author{
Lorena Itzel Noriega-Aguirre, ${ }^{*}$ Joel Méndez, ${ }^{\ddagger}$ Amny Trujillo* \\ *Centro de Diagnóstico y Tratamiento de las Enfermedades Respiratorias (CEDITER); ‡Universidad Especializada de las Américas \\ (UDELAS). Ciudad de Panamá, Panamá.
}

\begin{abstract}
RESUMEN. Objetivo: Determinar la prevalencia y las características de la enfermedad pulmonar obstructiva crónica (EPOC) en pacientes con síntomas respiratorios en la República de Panamá. Material y métodos: Se realizó un estudio en población general, mediante un muestreo por conglomerado en todas las provincias de la República de Panamá; en mayores de 40 años fue aplicado un cuestionario de síntomas respiratorios y se realizó una espirometría simple con broncodilatador a los que resultaron sintomáticos. Resultados: Aplicamos 877 encuestas en mayores de 40 años en las 10 provincias de la República de Panamá; 39 participantes presentaron síntomas y espirometría de EPOC, correspondiendo a una prevalencia global para Panamá, en sintomáticos respiratorios, de $4.4 \%$ (IC 3.1-5.8), 56.4\% (IC 40.8-72.0) del sexo masculino, 82.1\% (IC 70.194.1) no tenía diagnóstico previo de EPOC, y sólo 20.5\% (IC 7.83-33.2) se había realizado previamente una espirometría; 43.6\% (IC 28.0-59.1) tenía historia de tabaquismo positivo y $30.7 \%$ (IC 16.2-45.2) de exposición a biomasa; $28.2 \%$ (11/39) corresponden a estadío GOLD A, 38.5\% (15/39) a GOLD B, 2.5\% (1/39) a GOLD C y $30.8 \%$ (12/39) corresponden a GOLD D. Las comorbilidades más frecuentes fueron: rinitis en $38.4 \%$ (IC 23.2-53.7), hipertensión arterial en 35.9\% (IC 20.8-51.0) y síntomas de reflujo gastroesofágico en 23.1\% (IC 9.9-36.3). Los anticolinérgicos de acción prolongada fueron los fármacos más utilizados. Conclusiones: La prevalencia de EPOC en sintomáticos respiratorios en Panamá es baja, se presenta principalmente en el sexo masculino; el tabaquismo y la biomasa son las exposiciones más frecuentes. La espirometría para su diagnóstico está subutilizada. Las comorbilidades más frecuentes fueron rinitis, hipertensión arterial y reflujo gastroesofágico. Dos terceras partes de las EPOC correspondieron a los grupos B y D de GOLD, que son los más sintomáticos. Los anticolinérgicos de acción prolongada son los fármacos más utilizados y los esteroides inhalados se usaron en todos los grupos GOLD.
\end{abstract}

Palabras clave: Enfermedad pulmonar obstructiva crónica, biomasa, prevalencia de tabaquismo, Panamá.

\begin{abstract}
Objective: To determine the prevalence and characteristics of chronic obstructive pulmonary disease (COPD), in the Panama Republic. Material and methods: A prevalence study was carried out in the Republic of Panama, in subjects over 40 years of age, who answered affirmatively to the survey of respiratory symptoms, and in whom the diagnosis of COPD was confirmed, after a clinical evaluation and spirometry study. Results: 877 surveys were applied to people over 40 years of age in the 10 provinces of the Republic of Panama. 39 participants presented COPD symptoms and spirometry, corresponding to a global prevalence for Panama of $4.4 \% \mathrm{Cl}(3.1-5.8)$. $56.4 \% \mathrm{Cl}(40.8-72.0)$ were male. $82.1 \% \mathrm{Cl}(70.1-94.1)$ had no previous diagnosis of COPD and $20.5 \%$ $\mathrm{Cl}$ (7.83-33.2) had previously performed spirometry. The $43.6 \% \mathrm{Cl}(28.0-$ 59.1) had positive smoking and $30.7 \% \mathrm{Cl}(16.2-45.2)$ had a history of exposure to biomass. The $46.1 \% \mathrm{Cl}(30.4-61.7)$ was classified in GOLD group $\mathrm{B}, 28 \% \mathrm{Cl}(13.9-42.0)$ in group $\mathrm{D}, 20.5 \% \mathrm{Cl}(7.8-33.1)$ in group $\mathrm{A}$ and $5.1 \%$ in group $\mathrm{C}, \mathrm{Cl}$ (3.5-6.7). The most frequent comorbidities, rhinitis $38.4 \%$ Cl 95 (23.2-53.7), arterial hypertension 35.9\% Cl (20.8-51.0) and symptoms of gastroesophageal reflux $23.1 \% \mathrm{Cl}(9.9-36.3)$. The longacting anticholinergics were most frequent drugs used. Conclusions: The prevalence of COPD in Panama is low; it occurs mainly in males, smoking and exposure to biomass are the most frequent exposures. Spirometry for its diagnosis is underused. The most frequent comorbidities were rhinitis, arterial hypertension and gastroesophageal reflux. Two thirds of COPD correspond to GOLD groups B and D, which are the most symptomatic, and the treatment they receive most frequently is based on long-acting anticholinergic bronchodilators.
\end{abstract}

Keywords: Chronic obstructive pulmonary disease, biomass, smoking prevalence, Panama.

Correspondencia:

Dra. Lorena Itzel Noriega-Aguirre

Centro de Diagnóstico y Tratamiento de Enfermedades Respiratorias (CEDITER).

Correo electrónico: lonoriega@gmail.com; Inoriega@ceditersa.com

Recibido: 03-I-2021; aceptado: 07-VI-2021.

Citar como: Noriega-Aguirre LI, Méndez J, Trujillo A. Prevalencia y características de la enfermedad pulmonar obstructiva crónica en la República de Panamá. Neumol Cir Torax. 2021; 80 (3): 173-178. https://dx.doi.org/10.35366/102477 


\section{INTRODUCCIÓN}

La enfermedad pulmonar obstructiva crónica (EPOC) se caracteriza por presentar síntomas respiratorios persistentes como tos, con o sin producción de esputo, y disnea. Estos síntomas son progresivos en el tiempo y en intensidad, y se producen debido a una limitación al flujo de aire. La espirometría es el estudio de función pulmonar por excelencia para el diagnóstico, ya que permite medir el grado de obstrucción. ${ }^{1}$

Para desarrollar EPOC, los principales factores de riesgos incluyen exposición a humo de tabaco y a material de biomasa (humo de leña), aunque existen también otros factores ambientales y genéticos. ${ }^{2}$

Hoy día, se considera a la EPOC como una enfermedad sistémica, asociada con otras enfermedades como las cardiovasculares, metabólicas y cáncer, ${ }^{3}$ cuyo tratamiento de base es el uso de medicamentos broncodilatadores inhalados para mejorar síntomas y calidad de vida. No hay tratamientos que curen o reviertan la enfermedad.

La prevalencia de la EPOC es variable y se estima globalmente en un 10\%. ${ }^{1}$ En Latinoamérica, de acuerdo con los datos del estudio Platino, la prevalencia varía entre $7.8 \%$ en México y $15 \%$ en Uruguay. ${ }^{4}$ En la actualidad, no contamos con datos previos sobre la prevalencia o características de pacientes con EPOC en la República de Panamá.

El objetivo del estudio fue determinar la prevalencia y características de la EPOC en pacientes mayores de 40 años con síntomas respiratorios.

\section{MATERIAL Y MÉTODOS}

Aplicamos una encuesta a nivel nacional, en población general mayor de 18 años. La muestra se calculó utilizando la fórmula de tamaño de muestra para proporción con población de referencia conocida, usando un nivel de confianza de $99.99 \%$ y una precisión de $p=0.01$. El estudio abarcó las 10 provincias de la República de Panamá, que se detallan a continuación con sus respectivas capitales: Bocas del Toro (Changuinola), Colón (Colón), Chiriquí (David), Darién (La Palma), Coclé (Penonomé), Herrera (Chitré), Los Santos (Las Tablas), Panamá (Panamá), Panamá Oeste (La Chorrera) y Veraguas (Santiago).

Para asignar la muestra por provincias, utilizamos la distribución porcentual de la población por provincia, en relación con la población total de la República de Panamá, de acuerdo con las estimaciones del Instituto Nacional de Estadísticas y Censo (INEC). ${ }^{5}$ La muestra fue distribuida por provincias de la siguiente manera: Panamá 41\%, Panamá Oeste $15 \%$, Chiriquí $12 \%$, Colón $7.4 \%$, Coclé $6.9 \%$, Veraguas $6.4 \%$, Bocas del Toro $4.3 \%$, Herrera $3.1 \%$, Los Santos $2.5 \%$ y Darién $1.4 \%$.

Fueron realizados 1,802 contactos telefónicos por medios electrónicos, aplicándose 1,532 encuestas y obteniéndose 1,514 respuestas válidas para análisis. Este es un subanálisis del estudio de prevalencia de enfermedades respiratorias obstructivas en Panamá (estudio PRENFOR), 6,7 para el cual se seleccionaron las encuestas realizadas en mayores de 40 años, que fueron 877.

La encuesta incluyó preguntas sobre diagnóstico previo de asma, EPOC, bronquitis crónica o enfisema, indicados por un médico; historia de sibilancias, opresión torácica, visitas al cuarto de urgencia en el último año, sensación de falta de aire, uso de medicamentos inhalados, opresión torácica o bronquitis con los resfriados, historia pasada o actual de tabaquismo y exposición a humo de leña (estudio PRENFOR). ${ }^{6,7}$

Los participantes que contestaron afirmativamente fueron invitados a una evaluación médica, en la cual, además de la historia clínica detallada, se realizó espirometría simple y con broncodilatador, siguiendo los estándares de ATS/ERS. ${ }^{8}$

La definición de los casos con EPOC fue realizada según GOLD (Global Initiative for Chronic Obstructive Lung Disease), en todo paciente con historia de posible factores

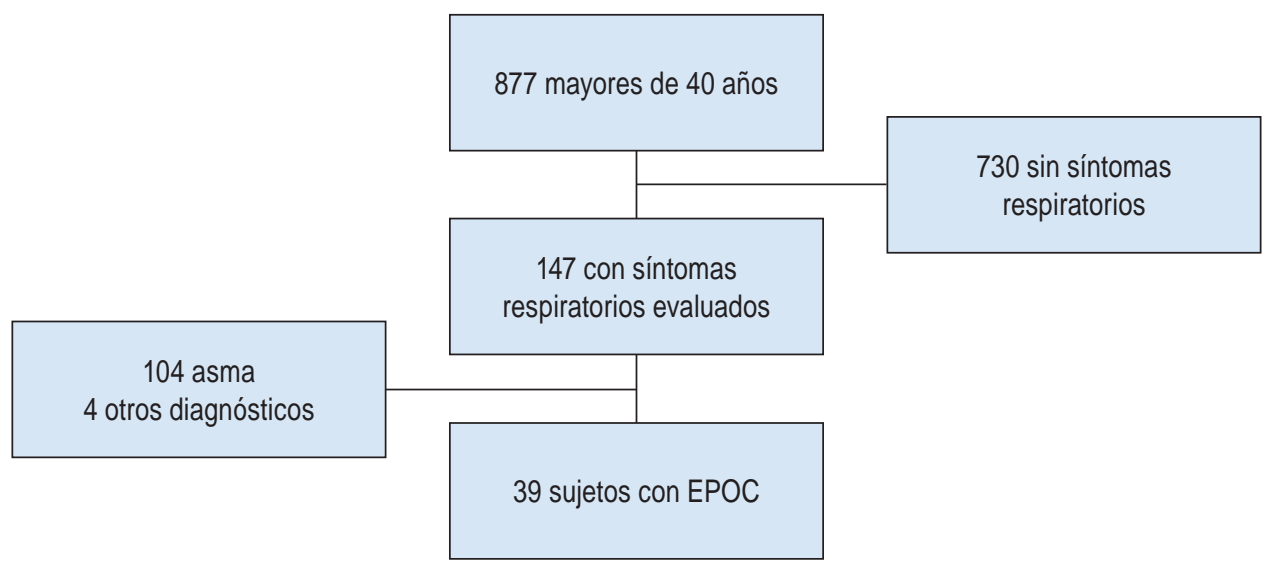

Figura 1:

Diagrama del estudio. $\mathrm{EPOC}=$ Enfermedad pulmonar obstructiva crónica. 
Tabla 1: Características de pacientes con EPOC en la República de Panamá.

\begin{tabular}{|l|c|c|c|c|}
\hline Característica & & $\mathrm{n}$ & $\%$ & IC (95\%) \\
\hline Sexo & Masculino & 22 & 56.4 & $40.8-72.0$ \\
& Femenino & 17 & 43.6 & $28.0-59.2$ \\
\hline Historia previa de EPOC & Sí & 7 & 17.9 & $5.9-30.0$ \\
\hline Espirometría previa & Sí & 8 & 20.5 & $7.8-33.2$ \\
\hline Media de FEV1/FVC & & & 63.4 & \\
\hline FEV1 posbroncodilatador & Leve $>80 \%$ & 1 & 2.6 & $-2.4-7.5$ \\
& Moderada 80-50\% & 23 & 59.0 & $43.5-74.4$ \\
& Grave 49-30\% & 13 & 33.3 & $18.5-48.1$ \\
\hline Muy grave <30\% & 2 & 5.1 & $-1.8-12.1$ \\
\hline Exposición a biomasa & Sí & 17 & 43.6 & $28.0-59.2$ \\
\hline Drogas inhaladas & Sí & 12 & 30.7 & $16.3-45.3$ \\
\hline \multirow{2}{*}{ Índice de disnea mMRC } & Sí & 1 & 2.6 & $-2.4-7.5$ \\
\hline Cuestionario CAT & $0-1$ & 15 & 38.4 & $23.2-53.7$ \\
\hline Índice de masa corporal & $\geq 2$ & 24 & 61.6 & $46.3-76.8$ \\
\hline Exacerbaciones en 12 meses & $>10$ & 27 & 69.2 & $54.7-83.7$ \\
& $<10$ & 12 & 30.8 & $16.3-45.3$ \\
\hline Hospitalizaciones en 12 meses & $>21$ & 32 & 82.1 & $70.0-94.1$ \\
& $>1$ & 7 & 17.9 & $5.9-30.0$ \\
\hline
\end{tabular}

EPOC $=$ enfermedad pulmonar obstructiva crónica; FEV1 = volumen espiratorio forzado en el 1 segundo; $F V C=$ capacidad vital forzada; $\mathrm{mMRC}=$ escala del Medical Research Council modificada; CAT = COPD Assessment Test.

de riesgo como humo de tabaco, biomasa o factores ambientales que presentara tos y disnea crónica y progresiva, con o sin sibilancias al examen físico y espirometría con patrón obstructivo parcialmente reversible, definido por la relación del volumen espiratorio en el primer segundo (FEV1) sobre la capacidad vital forzada (FVC) menor de $70 \%$, (FEV1/FVC < de 0.7) posbroncodilatador.

En el análisis de datos, las variables cualitativas del estudio fueron presentadas en frecuencias absolutas con intervalos de confianza a 95\%. Las variables cuantitativas se presentaron en frecuencias absolutas con desviación estándar como medida de dispersión. Todos los datos fueron ingresados en una base de datos con el programa Epi Info versión 7.1.

El estudio fue realizado cumpliendo normativas internacionales de Buenas Prácticas Clínicas, revisado y aprobado por el Comité de Bioética en Investigación del Hospital Punta Pacífica. El consentimiento informado fue obtenido de forma verbal para la encuesta, y de forma escrita para la evaluación clínica y espirométrica.

\section{RESULTADOS}

Evaluamos 877 encuestas en mayores de 40 años, 147 presentaron síntomas respiratorios, luego de la evaluación clínica y estudio de espirometría calificaron como EPOC 39 sujetos, lo que nos da una prevalencia global para Panamá de EPOC en sintomáticos respiratorios de 4.4\% (IC 3.1-5.8) (Figura 1).

La Tabla 1 muestra las características de los pacientes con diagnóstico de EPOC, donde 56.4\% (IC 40.8-72.0) (22/39) corresponde al sexo masculino. En 82.1\% (IC 70.194.1) (32/39) no se conocía diagnóstico previo de EPOC y 20.5\% (IC 7.83-33.2) de los casos (8/39) tenía algún estudio previo de espirometría.

Con relación a los antecedentes de exposición a tabaco, 43.6 \% (IC 28.0-59.1) (17/39) tiene historia de tabaquismo positivo, con un promedio del índice paquete-año de 25.82 , con un rango de 4 a 100 paquetes-año, y 30.7\% (IC 16.2-45.2) (12/39) tiene historia de exposición a biomasa. Sólo 2.5\% (IC 2.4-7.4) (1/39) reportó uso de otras drogas ilícitas inhaladas. 
Cuando evaluamos las exacerbaciones, el índice de disnea de Medical Research Council modificado (mMRC) ${ }^{9} \mathrm{y}$ el cuestionario COPD Assessment Test ${ }^{10}$ (CAT por sus siglas en inglés), para clasificar los casos de acuerdo con GOLD, encontramos que 28.2\% (11/39) corresponden a GOLD A, $38.5 \%(15 / 39)$ a GOLD B, $2.5 \%(1 / 39)$ a GOLD C y $30.8 \%$ (12/39) corresponden a GOLD D. El grupo con una mayor cantidad de exacerbaciones fue el D con 54 eventos en un año (Tabla 2).

Las comorbilidades encontradas en los pacientes con EPOC se muestran en la Tabla 3, siendo las más frecuentes rinitis en 38.4\% (IC 23.2-53.7) (15/39), hipertensión arterial $35.9 \%$ (IC 20.8-51.0) (14/39) y síntomas de reflujo gastroesofágico en 23.1\% (IC 9.9-36.3) (9/39).

Con relación a los medicamentos utilizados para tratamiento de la EPOC por grupo GOLD, encontramos que el uso de anticolinérgicos de acción prolongada (LAMA, por sus siglas en inglés) fueron los fármacos más utilizados: en $54.5 \%(6 / 11)$ de los clasificados como GOLD A, 66.7\% (10/15) de los GOLD B y 66.7\% (8/12) de los GOLD D los utilizaban.

Los esteroides inhalados fueron el segundo grupo de medicamentos más utilizados: en $27.3 \%(3 / 11)$ de los GOLD A, 46.7\% (7/15) de los GOLD B y 33.3\% (4/11) de los GOLD D (Tabla 4).

\section{DISCUSIÓN}

Encontramos una prevalencia de EPOC en pacientes sintomáticos respiratorios en Panamá de 4.4\% (IC 3.1-5.8), este es el primer dato de prevalencia para esta enfermedad en nuestro país, por lo que no contamos con valores previos para comparar. En cuanto a la región latinoamericana, corresponde a una de las prevalencias más bajas, considerando que varían entre un $6.2 \%$ en Barranquilla, Colombia en 2008, y $19.7 \%$ en Montevideo, Uruguay en 2004. ${ }^{4}$ También corresponde a una prevalencia baja si la comparamos con la prevalencia global de EPOC, que se estima entre 7.6 y $10 \%{ }^{1,2}$

Dentro de los posibles factores que influyen en este valor, pudiéramos considerar las políticas antitabaco instauradas

Tabla 2: Clasificación basada en GOLD.

\begin{tabular}{|c|c|c|}
\hline $\begin{array}{c}\text { Exacerbaciones } \\
(\geq 2)\end{array}$ & $\mathrm{C}$ & $\mathrm{D}$ \\
& $2.5 \%(1)$ & $30.8 \%(12)$ \\
\hline $\begin{array}{c}\text { Exacerbaciones } \\
(0-1)\end{array}$ & $\mathrm{A}$ & $\mathrm{B}$ \\
$\mathrm{CAT}$ & $28.2 \%(11)$ & $38.5 \%(15)$ \\
\hline $\mathrm{mMRC}$ & $<10$ & $\geq 10$ \\
\hline
\end{tabular}

GOLD = Global Initiative for Chronic Obstructive Lung Disease; CAT = COPD Assessment Test; $\mathrm{mMRC}=$ escala del Medical Research Council modificada.
Tabla 3: Morbilidades de pacientes con EPOC en la República de Panamá.

\begin{tabular}{|l|r|r|r|}
\hline Característica & $\mathbf{n}$ & $\%$ & IC (95\%) \\
\hline Síntomas de rinitis & 15 & 38.4 & $23.2-53.7$ \\
\hline Síntomas de reflujo & 9 & 23.1 & $9.9-36.3$ \\
\hline Hipertensión arterial & 14 & 35.9 & $20.8-51.0$ \\
\hline Cardiopatías & 4 & 10.3 & $0.7-19.8$ \\
\hline Diabetes & 2 & 5.1 & $-1.8-12.1$ \\
\hline Dislipidemia & 2 & 5.1 & $-1.8-12.1$ \\
\hline Cataratas & 4 & 10.3 & $0.7-19.8$ \\
\hline Prostatismo & 5 & 12.8 & $2.3-23.3$ \\
\hline Algún tipo de cáncer & 3 & 7.7 & $-0.7-16.1$ \\
\hline
\end{tabular}

EPOC $=$ enfermedad pulmonar obstructiva crónica .

en 2008, que hacen de Panamá un país referente en la región en medidas para el control del tabaco y, por ende, de sus consecuencias. ${ }^{11}$ Por otra parte, en esta investigación se buscaron sólo pacientes sintomáticos respiratorios, por lo que pudimos dejar de captar los casos más leves o asintomáticos, lo cual es un sesgo del estudio.

El diagnóstico de EPOC fue más prevalente en el sexo masculino, igual que lo descrito en otras series. ${ }^{12,13}$ En el grupo estudiado encontramos un subdiagnóstico de EPOC de $80 \%$, estos datos son similares a los reportes en otras publicaciones; ${ }^{12,13}$ este hecho preocupa, dado que son pacientes que se mantienen sin tratamiento a pesar de tener la enfermedad, lo que hace necesario tener estrategias para diagnósticos más tempranos de la EPOC, como sería la búsqueda activa en grupos con factores de riesgo, en la red de atención primaria, con cuestionarios dirigidos a fumadores y/o expuestos a humo de leña o biomasa. ${ }^{14-16}$

Por otra parte, el diagnóstico de EPOC se basa principalmente en la medición de flujo de aire mediante la espirometría, en nuestro estudio sólo un $20 \%$ de los casos con síntomas respiratorios que resultaron con EPOC tenían una espirometría previa, lo que nos muestra la subutilización de esta importante herramienta diagnóstica, una razón local puede ser que existen pocos equipos de espirometría funcionando en la red de atención primaria en el ámbito nacional, y suelen concentrarse en hospitales con servicios de neumología, similar a lo que ocurre en otros países. ${ }^{17}$

El mayor porcentaje de casos captados se encontraba en los grupos B y D de GOLD, que son pacientes con más síntomas respiratorios, esto puede corresponder a un sesgo, ya que la captación de sujetos se basó en la presencia de síntomas respiratorios en población general, no fue búsqueda dirigida a grupos de riesgo como fumadores, por ejemplo, ni espirometría en población asintomática al azar. 
Tabla 4: Uso de medicamentos en pacientes con EPOC tratados en la República de Panamá.

\begin{tabular}{|l|r|r|r|}
\hline Grupo de medicamento & \multicolumn{1}{c|}{$\mathbf{n}$} & \multicolumn{1}{c|}{$\%$} & IC (95\%) \\
\hline Pacientes con tratamiento previo & \multicolumn{1}{|c|}{26} & 100.0 & \\
\hline Beta 2 agonistas & & & \\
$\quad$ Corta acción (SABA) & 7 & 26.9 & $9.9-44.0$ \\
Acción prolongada (LABA) & 10 & 38.5 & $19.8-57.2$ \\
\hline Anticolinérgicos & & & \\
$\quad$ Corta acción (SAMA) & 8 & 30.1 & $13.0-48.5$ \\
Acción prolongada (LAMA) & 18 & 69.2 & $51.5-87.0$ \\
\hline Combinados LABA/LAMA & 5 & 19.2 & $4.1-34.4$ \\
\hline Esteroides inhalados & 9 & 34.1 & $16.3-52.9$ \\
\hline Combinados LABA/esteroides & 5 & 19.2 & $4.1-34.4$ \\
\hline
\end{tabular}

EPOC $=$ enfermedad pulmonar obstructiva crónica.

Los posibles factores de riesgos relacionados con la EPOC fueron el tabaquismo y la exposición a biomasa, similar a lo descrito en la literatura, ${ }^{2}$ y las comorbilidades más frecuentes en nuestra serie fueron rinitis y reflujo gastroesofágico, ${ }^{18}$ a diferencia de la literatura, donde se describen con mayor frecuencia las cardiovasculares, que se ubicaron en la tercera y cuarta posición en nuestros resultados. $2,18,19$

En cuanto a los grupos de medicamentos, los LAMA fueron los más utilizados; sin embargo, observamos el uso de esteroides inhalados como segundo grupo de fármacos más utilizados y en todos los grupos GOLD, no sólo en los exacerbadores, como indican las recomendaciones GOLD. ${ }^{1}$

\section{CONCLUSIONES}

La prevalencia de EPOC en sintomáticos respiratorios en Panamá fue de $4.4 \%$, principalmente del sexo masculino y más frecuente relacionado al hábito del tabaco. La espirometría, que corresponde al método estándar para su diagnóstico es subutilizada en nuestro país y sólo en uno de cada cinco se había realizado previamente esta prueba.

Las comorbilidades más frecuentes encontradas en pacientes con EPOC fueron rinitis, hipertensión arterial y reflujo gastroesofágico. Dos terceras partes de los EPOC captados en el estudio correspondieron a los grupos B y D de GOLD, que son los más sintomáticos, y el tratamiento que con mayor frecuencia reciben se basa en broncodilatadores anticolinérgicos de acción prolongada.

\section{Limitaciones}

La investigación cuenta con las limitaciones propias del diseño de los estudios de prevalencia; de manera adicional, muchos pacientes pueden minimizar los síntomas o relacionarlos con el envejecimiento, y se incluyeron sólo sintomáticos respiratorios, lo que pudo hacer que no captáramos casos asintomáticos con la encuesta.

Por otra parte, la cantidad de pacientes que resultaron con síntomas y espirometría compatible con EPOC corresponde a un número relativamente pequeño, lo que limita su caracterización, y sólo realizamos espirometría a aquéllos con síntomas respiratorios, no hubo búsqueda activa en casos con factores de riesgo.

\section{REFERENCIAS}

1. Global Initiative for chronic obstructive lung disease. Gold Reports [Internet]: Fontana; 2020. Available in: https://goldcopd.org/gold-reports/

2. Raherison C, Girodet PO. Epidemiology of COPD. Eur Respir Rev [Internet]. 2009;18(114):213-221. Available in: http://dx.doi. org/10.1183/09059180.00003609

3. Cavailles A, Brinchault-Rabin G, Dixmier A, Goupil F, Gut-Gobert C, Marchand-Adam S, et al. Comorbidities of COPD. Eur Respir Rev. 2013;22(130):454-475. doi: 10.1183/09059180.00008612.

4. Menezes AM, Perez-Padilla R, Jardim JR, Muiño A, Lopez MV, Valdivia G, et al. PLATINO Team. Chronic obstructive pulmonary disease in five Latin American cities (the PLATINO study): a prevalence study. Lancet [Internet]. 2005;366(9500):1875-1881. Available in: https://doi. org/10.1016/S0140-6736(05)67632-5

5. Instituto Nacional de Estadísticas y Censo. INEC [Internet]: Panamá; 2012. Disponible en: https://www.inec.gob.pa/publicaciones/

6. Noriega L, Méndez J. Prevalencia de síntomas respiratorios de enfermedades respiratorias obstructivas, asma y EPOC en mayores de 18 años en la República de Panamá. RMP. 2020;40(2):64-69. Disponible en: http://dx.doi.org/10.37980/im.journal.rmdp.2020873

7. Noriega L, Méndez J, Trujillo A, Aguilera A, García Y. Prevalencia y características del asma en mayores de 18 años en la República de Panamá: estudio de base poblacional PRENFOR. Open Respiratory Archives. 2020. Disponible en: http://doi.org/10.1016/j. opresp.2020.04.002

8. Miller MR, Hankinson J, Brusasco V, Burgos F, Casaburi R, Coates A, et al. Standardisation of spirometry. Eur Respir J. 2005;26(2):319338. doi: 10.1183/09031936.05.00034805.

9. Perez T, Burgel PR, Paillasseur JL, Caillaud D, Deslée G, Chanez P, et al. Modified medical research council scale vs baseline dyspnea index to evaluate dyspnea in chronic obstructive pulmonary disease. Int J Chron Obstruct Pulmon Dis. 2015;10:1663-1672. doi: 10.2147/ COPD.S82408.

10. Jones PW, Harding G, Berry P, Wiklund I, Chen WH, Kline Leidy N. Development and first validation of the COPD assessment test. Eur Respir J. 2009;34(3):648-654. Available in: http://doi. org/10.1183/09031936.00102509

11. Ley No. 13Que adopta medida para el control del tabaco y sus efectos en la salud. Gaceta Oficial Digital Gobierno de la República de Panamá [en línea]. 2008 [Citado el 14 de septiembre de 2020]. Disponible en: https://www.gacetaoficial.gob.pa/pdfTemp/25966/8638.pdf 
12. Ntritsos G, Franek J, Belbasis L, Christou MA, Markozannes G, Altman $\mathrm{P}$, et al. Gender-specific estimates of COPD prevalence: a systematic review and meta-analysis. Int J Chron Obstruct Pulmon Dis. 2018;13:1507-1514. Available in: http://doi.org/10.2147/COPD.S146390

13. Lopez Varela MV, Montes de Oca M, Halbert RJ, Muiño A, PerezPadilla R, Tálamo C, et al. Sex-related differences in COPD in five Latin American cities: the PLATINO study. Eur Respir J. 2010;36(5):10341041. Available in: http://doi.org/10.1183/09031936.00165409

14. Lamprecht B, Soriano JB, Studnicka M, Kaiser B, Vanfleteren LE, Gnatiuc $L$, et al. Determinants of underdiagnosis of COPD in national and international surveys. Chest. 2015;148(4):971-985. Available in: http://doi.org/10.1378/chest.14-2535

15. Ancoechea J, Badiola C, Duran-Taulería E, García Río F, Miratvilles M, Muñoz L, et al. Estudio EPI-SCAN: resumen del protocolo de un estudio para estimar la prevalencia de EPOC en personas de 40 a 80 años en España. Arch Bronconeumol. 2009;45:41-47. Disponible en: http://doi.org/10.1016/j.arbres.2008.06.001

16. Schiavi E, Stirbulov R, Hernández Vecino R, Mercurio S, Di Boscio V; Puma Team. COPD screening in primary care in four
Latin American countries: methodology of the PUMA Study. Arch Bronconeumol. 2014;50(11):469-74. Available in: http://doi. org/10.1016/j.arbres.2014.03.006

17. Laniado-Laborín R, Rendón A, Alcantar-Schramm JM, CazaresAdame R, Bauerle O. Subutilization of COPD guidelines in primary care: a pilot study. J Prim Care Community Health. 2013;4(3):172-176. Available in: http://doi.org/10.1177/2150131913475817

18. Lee AL, Goldstein RS. Gastroesophageal reflux disease in COPD: links and risks. Int J Chron Obstruct Pulmon Dis. 2015;10:1935-1949. Available in: http://doi.org/10.2147/COPD.S77562

19. Negewo NA, Gibson PG, McDonald VM. COPD and its comorbidities: impact, measurement and mechanisms. Respirology. 2015;20(8):11601171. Available in: http://doi.org/10.1111/resp.12642

Fuentes de financiamiento: El presente trabajo ha sido financiado por el programa Investigator Initiated Trials de Novartis, donde el diseño del estudio, la ejecución del proyecto y análisis de datos estuvo a cargo del equipo investigador. 